\title{
Mycobacterium canariasense sp. nov.
}

\author{
Correspondence \\ M. Soledad Jiménez \\ msjimenz@isciii.es
}

\author{
M. Soledad Jiménez, ${ }^{1}$ M. Isolina Campos-Herrero, ${ }^{2}$ Diana García, ${ }^{2}$ \\ Marina Luquin, ${ }^{3}$ Laura Herrera ${ }^{1}$ and María J. García ${ }^{4}$ \\ ${ }^{1}$ Laboratorio de Micobacterias, Servicio de Bacteriología, Centro Nacional de Microbiología, \\ Instituto de Salud Carlos III, Majadahonda, Madrid, Spain \\ ${ }^{2}$ Servicio de Microbiología, Hospital de Gran Canaria Dr Negrin, Las Palmas de Gran Canaria,
Spain \\ Unitat de Microbiología, Departament de Genetica i de Microbiología, Universitat Autónoma de \\ Barcelona, Bellaterra, Barcelona, Spain \\ ${ }^{4}$ Departamento de Medicina Preventiva, Facultad de Medicina, Universidad Autónoma de \\ Madrid, Madrid, Spain
}

\begin{abstract}
A novel rapidly growing, non-pigmented mycobacterium was isolated from blood samples obtained from 17 patients with febrile syndrome. Bacterial growth occurred at 30 and $37{ }^{\circ} \mathrm{C}$ on Löwenstein-Jensen medium and also on MacConkey agar without crystal violet. Strains contained $\alpha$ - and $\alpha^{\prime}$-mycolates in their cell wall. Sequence analysis of the $h s p 65$ and $16 S$ rRNA genes identified the isolates as rapidly growing mycobacteria. Sequences of both genes were unique within the mycobacteria. DNA-DNA hybridization showed that the isolates had less than $15 \%$ reassociation with 13 other recognized rapidly growing mycobacteria. The name Mycobacterium canariasense sp. nov. is proposed for this novel opportunistic pathogen, which is most closely related to Mycobacterium diernhoferi. The type strain is $502329^{\top}$ $\left(=\mathrm{CIP} 107998^{\top}=\right.$ CCUG $\left.47953^{\top}\right)$.
\end{abstract}

Rapidly growing mycobacteria are ubiquitous environmental bacteria commonly found in water and soil (BrownElliott \& Wallace, 2002). Members of this group, especially the three major species Mycobacterium fortuitum, Mycobacterium chelonae and Mycobacterium abscessus, have been reported as aetiological agents of a variety of infections, including bacteraemia and disseminated disease in patients with long-term venous catheters (Raad et al., 1991). They have also been involved in nosocomial outbreaks or pseudooutbreaks related to contamination of hospital water supplies and reagents (Ashford et al., 1997; Chadha et al., 1998; LaBombardi et al., 2002). In this study, a novel mycobacterium involved in nosocomial infection is described; the name proposed for this species is Mycobacterium canariasense sp. nov.

During the period January 2000 to September 2002, a mycobacterium that could not be identified by conventional

The GenBank/EMBL/DDBJ accession numbers for the 16S rRNA and hsp65 genes of Mycobacterium canariasense strain $502329^{\top}$ are AY255478 and AY255477, respectively.

Some characteristics of strain $502329^{\top}$ (Table A), TLC of methyl mycolates of $M$. canariasense strains and related Mycobacterium species (Fig. A), GC analysis of fatty acid methyl esters of strain $502329^{\top}$ (Fig. B), hybridization dot-blot assays (Fig. C) and DNA-DNA relatedness between strain $502329^{\top}$ and other Mycobacterium species (Table B) are available as supplementary material in IJSEM Online. procedures was isolated from blood specimens of 17 patients with suspected nosocomial acquisition. Patients were admitted to a tertiary care hospital in the Canary Islands, Spain. Most of them $(n=15)$ had a malignant disease and all of them carried at that time a central venous catheter. Mycobacteria were considered to be the cause of the febrile syndrome in 12 cases. Ten patients were treated with specific antibiotic therapy and the central catheter was removed in nine of them. Catheters were left in two patients, who later relapsed. The same mycobacterium grew in their subsequent blood cultures. Most of the patients recovered after treatment. However, two patients died as a consequence of their disease status. All bacterial isolates had homogeneous biochemical characteristics and antimicrobial susceptibility patterns. The first isolate and four other randomly selected strains were sent to the reference Laboratory of Mycobacteria (Centro Nacional de Microbiología, Madrid, Spain) for identification. These five isolates were examined for several phenotypic and genotypic characteristics and results were compared with those displayed by other rapidly growing mycobacterial species.

Acid-alcohol-fastness, colony morphology and pigment production, as well as the ability to grow at various temperatures $\left(22-45^{\circ} \mathrm{C}\right)$, on Löwenstein-Jensen medium (LJ), in the presence of $5 \% \mathrm{NaCl}$ and on MacConkey agar without crystal violet (Kubica \& David, 1980) were examined. A total of 12 biochemical tests was performed 
(Marks \& Trollope, 1960), including the use of single carbon sources and acid formation (Silcox et al., 1981; Tsukamura, 1967). Hydrolysis of seven different amides was also tested (Vestal, 1975). Sensitivities to eight different antimycobacterial drugs were tested by the proportion method in LJ (Canetti et al., 1963). E-test strips on Mueller-Hinton medium were used to determine sensitivity to 13 further antibiotics.

Bacterial cells were partially acid-fast rods that grew on LJ, initially as non-pigmented colonies, but later developing a more yellowish, smooth, moist and shiny appearance. Growth on LJ occurred after $2-3$ days at 30 and $37^{\circ} \mathrm{C}$; growth did not occur at the other temperatures tested. The main phenotypic characteristics of isolates are indicated in Table 1 (see supplementary material in IJSEM Online for a complete set of data). All isolates of $M$. canariasense exhibited identical characteristics. The novel species could be differentiated from the $M$. chelonae group by single carbon source testing, from $M$. fortuitum and $M$. diernhoferi by the nitrate reduction test and from Mycobacterium frederiksbergense and other related species by pigmentation development (Table 1).

Fatty and mycolic acids were liberated from each strain sample by saponification. These compounds were then extracted with diethyl ether and treated with diazomethane to obtain the methyl esters (Daffé et al., 1983). Mycolic acid methyl esters were studied by analytical one-dimensional TLC using silica gel 60 TLC plates (Merck) as described previously (Luquín et al., 1991a). Fatty acid methyl esters and methyl mycolate cleavage products were determined by GC-MS (Luquin et al., 1991b).

TLC analysis of the mycolic acid methyl esters revealed that the five M. canariasense strains contained $\alpha$ - and $\alpha^{\prime}$ mycolates (see supplementary material available in IJSEM Online). To date, this mycolate pattern has only been described in M. chelonae and M. abscessus (see Table 1) (Hinrikson \& Pfyffer, 1994) and species within the genus Tsukamurella (Hamid et al., 1993). This mycolate pattern allows $M$. canariasense to be differentiated from related

Table 1. Phenotypic characteristics of $M$. canariasense and other rapidly growing mycobacteria

Species: 1, M. canariasense; 2, M. abscessus; 3, M. chelonae; 4, M. fortuitum; 5, M. mucogenicum; 6, M. senegalense; 7, M. immunogenum; 8, M. neoaurum; 9, M. diernhoferi; 10, M. frederiksbergense. -, Less than $15 \%$ of isolates are positive; + , more than $85 \%$ of isolates are positive; numbers in parentheses indicate percentages of positive strains; ND, not determined. Biochemical data corresponding to mycobacteria other than M. canariasense were taken from Schröder et al. (1997), Springer et al. (1995), Wayne \& Kubica (1986), Willumsen et al. (2001) and Wilson et al. (2001).

\begin{tabular}{|c|c|c|c|c|c|c|c|c|c|c|}
\hline Characteristic & 1 & 2 & 3 & 4 & 5 & 6 & 7 & 8 & 9 & 10 \\
\hline Pigmentation & - & - & - & - & - & - & - & + & - & + \\
\hline MacConkey agar & + & + & + & + & ND & ND & + & - & ND & - \\
\hline Growth on $5 \% \mathrm{NaCl}$ & - & + & - & + & - & + & - & + & + & $\mathrm{ND}$ \\
\hline Nitrate reductase & - & - & $(17)$ & + & $(45)$ & - & - & $(80)$ & + & + \\
\hline Tween hydrolysis & + & - & - & - & $\mathrm{ND}$ & ND & ND & $(60)$ & - & + \\
\hline Arylsulfatase (3 days) & + & + & + & + & $(84)$ & + & + & - & - & ND \\
\hline Iron uptake & - & - & - & + & - & - & - & + & ND & ND \\
\hline \multicolumn{11}{|c|}{ Use as sole carbon source: } \\
\hline Citrate & - & - & + & - & + & + & - & + & ND & ND \\
\hline D-Mannitol & + & - & - & - & + & + & - & + & + & ND \\
\hline i-myo-Inositol & + & - & - & - & - & ND & - & + & + & ND \\
\hline \multicolumn{11}{|l|}{ Acid production from: } \\
\hline Arabinose & + & - & - & - & ND & ND & ND & $(80)$ & + & ND \\
\hline Xylose & + & - & - & - & ND & ND & ND & $(80)$ & + & + \\
\hline Dulcitol & - & - & - & - & ND & ND & ND & - & ND & ND \\
\hline Allantoinase & + & - & - & + & ND & + & $\mathrm{ND}$ & + & + & $\mathrm{ND}$ \\
\hline Benzamidase & - & - & - & - & ND & + & ND & - & - & ND \\
\hline iso-Nicotinamidase & - & - & - & - & $\mathrm{ND}$ & + & $\mathrm{ND}$ & - & - & $\mathrm{ND}$ \\
\hline Succinidamidase & - & - & - & - & $\mathrm{ND}$ & $\mathrm{ND}$ & ND & - & - & - \\
\hline Mycolic acid type $\dagger$ & I, II & I, II & I, II & I, II $\ddagger, \mathrm{V}, \mathrm{VII} \ddagger$ & I, VI & I, II $\ddagger, V$, VII $\ddagger$ & $\mathrm{ND}$ & $\mathrm{I}, \mathrm{IV}, \mathrm{VI}$ & I, IV, VI & I, IV, VI \\
\hline
\end{tabular}

${ }^{\star}$ MacConkey agar without crystal violet.

$\dagger$ Mycolic acid type: I, $\alpha$-mycolate; II, $\alpha^{\prime}$-mycolate; III, methoxy-mycolate; IV, keto-mycolate; V, epoxy-mycolate; VI, was esters; VII, $\omega$-1 methoxy-mycolate.

¥May not be present in certain strains. 
species such as Mycobacterium mucogenicum and $M$. diernhoferi (see Table 1; Muñoz et al., 1997). GC-MS analysis of $M$. canariasense strains indicated the presence of fatty acid methyl esters with 14-24 carbon atoms, of which hexadecanoate $\left(C_{16: 0}\right)$ and octadecenoate $\left(C_{18: 1}\right)$ were the most prominent. All strains also showed appreciable amounts of tuberculostearate. The primary methyl ester derived from thermal cleavage of methyl mycolates was tetracosanoate $\left(\mathrm{C}_{24: 0}\right.$; see supplementary material available in IJSEM Online), which allows $M$. canariasense to be differentiated from Tsukamurella. Species of this genus released $\mathrm{C}_{20}$ and $\mathrm{C}_{22}$ esters from pyrolysis of mycolates, but not $\mathrm{C}_{24}$ (Goodfellow et al., 1978). Unlike M. canariasense, strains of $M$. diernhoferi, $M$. frederiksbergense, M. mucogenicum and Mycobacterium neoaurum revealed secondary alcohols by GC-MS analysis; thus, the absence of these compounds in $M$. canariasense strains enables them to be clearly differentiated from these closely related species.

PCR was performed on DNA isolated from bacterial LJ cultures using the boiling method and centrifugation. A 439 bp region of the $h s p 65$ gene was subjected to PCR amplification followed by restriction analysis using the primers and conditions described by Telenti et al. (1993). Amplicons were also sequenced in triplicate according to Ringuet et al. (1999) using the BigDye Terminator sequencing kit and the ABI Prism 3700 automated sequencer (Applied Biosystems). In addition, $1514 \mathrm{bp}$ of the 16S rRNA gene were sequenced from PCR amplicons produced as described by Springer et al. (1995). Sequences of $h s p 65$ and 16S rRNA genes were aligned against previously described sequences from rapidly growing mycobacterial species, using EDITSEQ and MEGALIGN software (Lasergene MegAlign; DNAstar) (Altschul et al., 1997). Sequences were clustered using CLUSTAL W and weightings were used to construct a phylogenetic dendrogram (Kimura, 1980).

The hsp65 gene of $M$. canariasense strains contained a PCR-RFLP pattern that differed from those published previously or compiled in the PRAsite database (http:// www.hospvd.ch:8005). Patterns consisted of two fragments of 325 and 130 bp by BstEII digestion and three fragments of 140, 90 and 80 bp by HaeIII digestion. The sequence of the $M$. canariasense hypervariable region (Ringuet et al., 1999) was significantly different from those of other closely related rapidly growing species. Phylogenetic analysis of $441 \mathrm{bp}$ of this gene demonstrated that $M$. diernhoferi and M. mucogenicum are the closest non-pigmented relatives (94.3 and $93.5 \%$ similarity, respectively), whereas $M$. neoaurum and $M$. frederiksbergense were the most closely related pigmented species $(94.4$ and $93.6 \%$ similarity, respectively) (Fig. 1).

Sequences of the $16 \mathrm{~S}$ rRNA gene were also identical in all five novel isolates studied. A detailed comparison of the $16 \mathrm{~S}$ rRNA gene sequence with sequences of other rapidly growing mycobacterial species demonstrated $100 \%$ similarity in the genus-specific regions, but revealed a significant number of differences in helix 18 and the species-specific regions (namely hypervariable regions B and A, respectively) (Kirschner et al., 1993). The complete sequence of the $16 \mathrm{~S}$ rRNA gene of $M$. canariasense shows substantial differences when compared with those corresponding to other rapidly growing mycobacteria. Fig. 2 shows a comparison of the $M$. canariasense $16 \mathrm{~S}$ rRNA gene sequence with 10 other closely related mycobacteria; Tsukamurella paurometabola was used as an outgroup. The dendrogram shows that $M$. diernhoferi is the closest non-pigmented relative $(99 \cdot 2 \%$ similarity) to $M$. canariasense, followed by $M$. mucogenicum $(97 \cdot 0 \%)$ and, as seen previously with the $h s p 65$ gene, M. neoaurum was the most closely related pigmented rapidly growing mycobacteria $(98.7 \%)$. The similarity between M. canariasense and T. paurometabola was $91 \cdot 2 \%$.

Total genomic DNA was purified from liquid bacterial cultures, as described previously (Domenech et al., 1994). DNA-DNA hybridization experiments were performed on membrane filters, using a novel dot-blot-based procedure. Genomic DNA from M. canariasense strain $502329^{\mathrm{T}}$

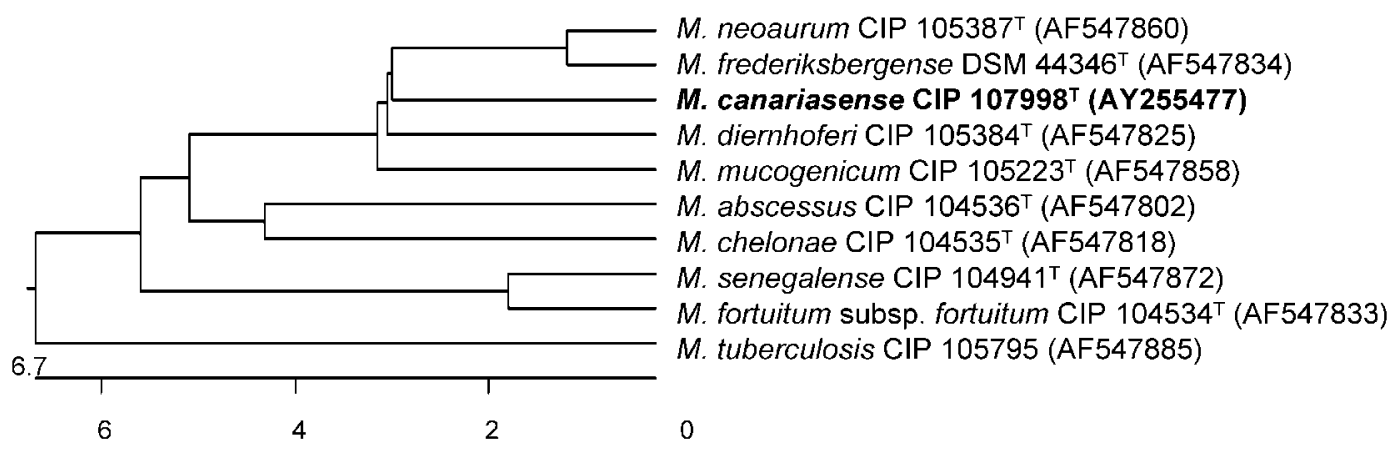

Fig. 1. Phylogenetic position of $M$. canariasense among other closely related, rapidly growing mycobacterial species as determined by $h s p 65$ gene sequences using the CLUSTAL W method with weightings. M. tuberculosis was used as outgroup. GenBank accession numbers are given in parentheses. 


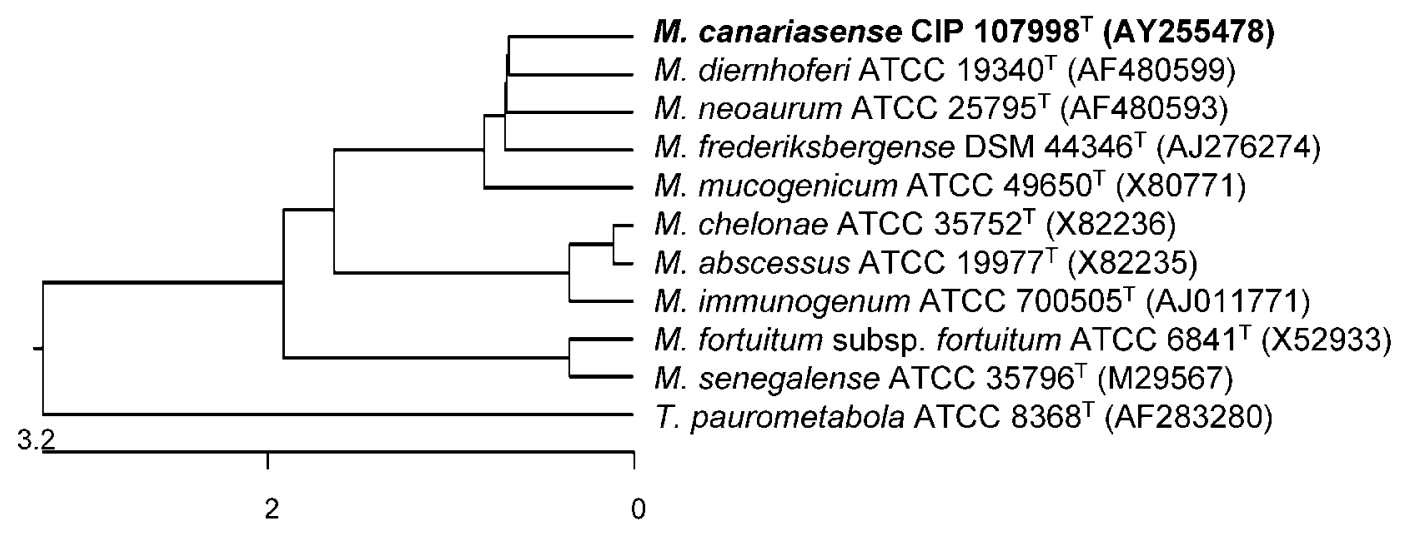

Fig. 2. Phylogenetic position of $M$. canariasense among other related rapidly growing mycobacterial species as determined by comparison of the 16S rRNA gene sequences. The tree was inferred using the CLUSTAL W method with weightings and rooted using T. paurometabola as outgroup. GenBank accession numbers are given in parentheses.

(300 ng) was labelled in vitro using the Megaprime DNA labelling system (Amersham) and $25 \mu \mathrm{Ci}(925 \mathrm{kBq})$ $\left[\alpha^{-}{ }^{32} \mathrm{P}\right] \mathrm{dCTP}$ (Amersham). Portions (500 ng) of each unlabelled DNA were dot-blotted and bound to nylon membrane filters (Hybond-N + ; Amersham). Hybridization and washes were carried out as described previously (Brown et al., 1999), except that ECL buffer (Amersham) was used both for pre-hybridization and hybridization steps. The relative binding ratios (expressed as percentages) for each species were calculated from the counts of homologous bound DNA, as measured using an Instant Imager counter (Izasa Instruments). In addition, the blots were stripped and rehybridized with a PCR amplicon from the $16 \mathrm{~S}$ rRNA gene as the new probe, as described by Domenech et al. (1997). This procedure has several advantages compared to previous methods (Keswani \& Whitman, 2001), mainly because the amount of DNA fixed in each dot can be determined by measuring the amount of radioactivity when using the 16S rRNA gene as the second probe. Due to the high sequence conservation within mycobacterial species inherent within this probe (Menendez et al., 2002), the radioactivity level can be considered to be proportional to the amount of target molecules on the filter. The number of copies of the 16S rRNA gene was already known for the mycobacterial species included in this work (Menendez et al., 2002; M. J. Garcia, unpublished results). The levels of DNA-DNA hybridization between $M$. canariasense strains were greater than $90 \%$. On the other hand, the level of reassociation between the $M$. canariasense type strain and 13 other related rapidly growing species corresponded to less than $15 \%$. These results strongly suggest that our isolates represent a novel species (Lan \& Reeves, 2001). Results of DNA-DNA hybridization analysis are available as supplementary material in IJSEM Online.

RFLP analysis of the 16S rRNA gene was also performed to complement DNA-DNA hybridization data (Domenech et al., 1994, 1997; Brown et al., 1999). A previously described experimental procedure (Domenech et al., 1997) was used, with the exception that ECL buffer (Amersham) was used for both the pre-hybridization and hybridization steps. Fig. 3 shows the RFLP patterns from BamHI-digested

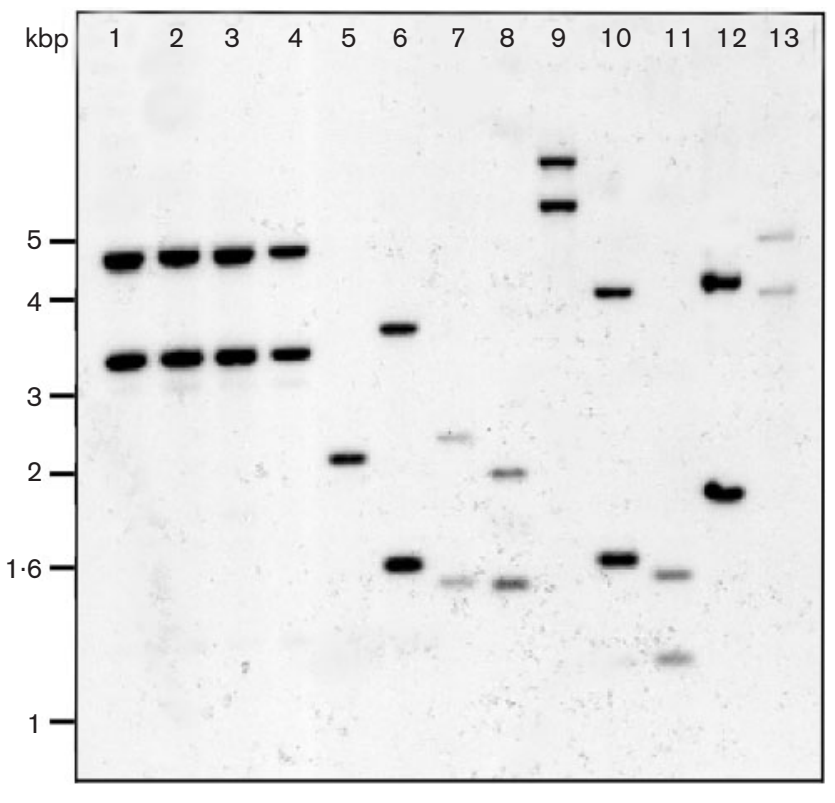

Fig. 3. RFLP patterns of $16 \mathrm{~S}$ rRNA genes from $M$. canariasense strains and other rapidly growing mycobacterial species. Patterns were obtained using $B a m H I$ as the restriction enzyme. Lanes: 1, M. canariasense $502329^{\top} ; 2$, M. canariasense $513578 ; 3, M$. canariasense $517062 ; 4, M$. canariasense 552822; 5, M. abscessus ATCC $19977^{\top} ; 6$, M. fortuitum subsp. fortuitum ATCC $6841^{\top} ; 7$, Mycobacterium goodii ATCC

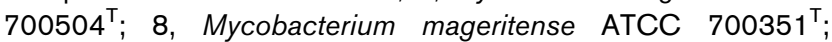
9, M. mucogenicum ATCC $49650^{\top} ; 10$, Mycobacterium porcinum CIPT $141460001=\mathrm{CIP} 105392^{\top} ; 11$, Mycobacterium smegmatis CIPT $141330010=\mathrm{CIP} 104444^{\top} ; 12$, Mycobacterium wolinskyi ATCC 700009; 13, N. asteroides ATCC 3308. Fragment sizes (in $\mathrm{kbp}$ ) are indicated on the left. 
genomic DNAs from M. canariasense strains, eight other rapidly growing mycobacterial species and Nocardia asteroides. Patterns of different species were different, but the patterns of all four M. canariasense strains were identical. All species tested, with the exception of $M$. abscessus, produced a pattern with two bands, indicating the presence of two copies of the 16S rRNA gene. These results indicate that $M$. canariasense belongs to the II-s mycobacterial class, i.e. species with two rrn operons per genome and short helix 18 in the $16 \mathrm{~S}$ rRNA gene coding region (Menendez et al., 2002).

On the basis of biochemical characteristics, mycolic and fatty acid patterns, PCR-RFLP of the $h s p 65$ gene, sequences of conserved genes and DNA-DNA hybridization, $M$. canariasense is proposed as a novel rapidly growing mycobacterium, most closely related to $M$. diernhoferi and M. chelonae.

\section{Description of Mycobacterium canariasense sp. nov.}

Mycobacterium canariasense (ca.na.ri.a.sen'se. L. gen. adj. canariasense referring to the Latin adjective of the Spanish islands where all strains were isolated).

Cells are partially acid-fast rods. Visible growth appears in 2-3 days as smooth, moist, shiny, non-pigmented colonies on Löwenstein-Jensen medium. Growth occurs at 30 and $37^{\circ} \mathrm{C}$, but not at 22,42 or $45^{\circ} \mathrm{C}$. Grows on MacConkey agar without crystal violet, but does not grow in the presence of $5 \% \mathrm{NaCl}$. Positive for arylsulfatase activity (3 days) and Tween 80 hydrolysis. Produces a low level of heatstable catalase and is negative for reduction of nitrates. These characteristics allow this novel species to be differentiated from other closely related species such as $M$. diernhoferi and M. mucogenicum. The inability to utilize citrate as a single carbon source allows differentiation from M. chelonae. Lipid composition of the cell wall is characterized by the presence of $\alpha$ - and $\alpha^{\prime}$-mycolates, similar to M. chelonae and M. abscessus. Results of DNA analyses such as PCR-RFLP of the hsp65 gene, sequences of conserved genes and DNA-DNA hybridization define $M$. canariasense as a distinct genomic mycobacterial species most closely related to $M$. diernhoferi and M. mucogenicum. The $16 \mathrm{~S}$ rRNA and $h s p 65$ gene sequences of $M$. canariasense are unique. This species belongs to the II-s mycobacterial class according to the classification of Menendez et al. (2002).

The type strain is $502329^{\mathrm{T}}\left(=\mathrm{CIP} 107998^{\mathrm{T}}=\mathrm{CCUG}\right.$ $\left.47953^{\mathrm{T}}\right)$.

\section{Acknowledgements}

We are grateful to Dr T. J. Bull for his helpful revision of the manuscript. This work was supported by grants from Spanish institutions (2002SGR-00099 from the Generalitat de Catalunya; and 08.2/0009/2001 from the Comunidad Autonoma de Madrid) and from the European Union (ICA4-CT-2001-10087).

\section{References}

Altschul, S. F., Madden, T. L., Schaffer, A. A., Zhang, J., Zhang, Z., Miller, W. \& Lipman, D. J. (1997). Gapped BLAST and PSI-BLAST: a new generation of protein database search programs. Nucleic Acids Res 25, 3389-3402.

Ashford, D. A., Kellerman, S., Yakrus, M., Brim, S., Good, R. C., Finelli, L., Jarvis, W. R. \& NcNeil, M. M. (1997). Pseudo-outbreak of septicemia due to rapidly growing mycobacteria associated with extrinsic contamination of culture supplement. J Clin Microbiol 35, 2040-2042.

Brown, B. A., Springer, B., Steingrube, V. A. \& 10 other authors (1999). Mycobacterium wolinskyi sp. nov. and Mycobacterium goodii sp. nov., two new rapidly growing species related to Mycobacterium smegmatis and associated with human wound infections: a cooperative study from the International Working Group on Mycobacterial Taxonomy. Int J Syst Bacteriol 49, 1493-1511.

Brown-Elliott, B. A. \& Wallace, R. J., Jr (2002). Clinical and taxonomic status of pathogenic non-pigmented or late-pigmenting rapidly growing mycobacteria. Clin Microbiol Rev 15, 716-746.

Canetti, G., Rist, N. \& Grosset, J. (1963). Mesure de la sensibilite du bacille tuberculeux aux drogues antibacillaires por le methode des proportions. Rev Tuberc Pneumol 27, 217-272.

Chadha, R., Grover, M., Sharma, A., Lakshmy, A., Deb, M., Kumar, A. \& Mehta, G. (1998). An outbreak of post-surgical wound infections due to Mycobacterium abscessus. Pediatr Surg Int 13, 406-410.

Daffé, M., Lanéelle, M. A., Asselineau, C., Lévy-Frebault, V. \& David, H. (1983). Intérêt taxonomique des acides gras des mycobactéries: proposition d'une methode d'analyse. Ann Microbiol (Paris) 134B, 241-256.

Domenech, P., Menendez, M. C. \& Garcia, M. J. (1994). Restriction fragment length polymorphisms of $16 \mathrm{~S}$ rRNA genes in the differentiation of fast-growing mycobacterial species. FEMS Microbiol Lett 116, 19-24.

Domenech, P., Jimenez, M. S., Menendez, M. C., Bull, T. J., Samper, S., Manrique, A. \& Garcia, M. J. (1997). Mycobacterium mageritense sp. nov. Int J Syst Bacteriol 47, 535-540.

Goodfellow, M., Orlean, P. A. B., Collins, M. D., Alshamaony, L. \& Minnikin, D. E. (1978). Chemical and numerical taxonomy of strains received as Gordona aurantiaca. J Gen Microbiol 109, 57-68.

Hamid, M. E., Minnikin, D. E. \& Goodfellow, M. (1993). A simple chemical test to distinguish mycobacteria from other mycolic-acidcontaining actinomycetes. J Gen Microbiol 139, 2203-2313.

Hinrikson, H. P. \& Pfyffer, G. E. (1994). Mycobacterial mycolic acids. Med Microbiol Lett 3, 49-57.

Keswani, J. \& Whitman, W. B. (2001). Relationship of $16 \mathrm{~S}$ rRNA sequence similarity to DNA hybridization in prokaryotes. Int J Syst Evol Microbiol 51, 667-678.

Kimura, M. (1980). A simple method for estimating evolutionary rates of base substitutions through comparative studies of nucleotide sequences. J Mol Evol 16, 111-120.

Kirschner, P., Meier, A. \& Böttger, E. C. (1993). Genotypic identification and detection of mycobacteria - facing novel and uncultured pathogens. In Diagnostic Molecular Microbiology. Principles and Applications, pp. 173-190. Edited by D. H. Persing, T. F. Smith, F. C. Tenover \& T. J. White. Washington, DC: American Society for Microbiology.

Kubica, G. P. \& David, H. L. (1980). The mycobacteria. In Gradwohl's Clinical Laboratory Methods and Diagnosis, pp. 1693-1730. Edited by A. C. Sonnenwirt \& L. Jaret. St Louis: C. V. Mosby.

LaBombardi, V. J., O’Brien, A. M. \& Kislak, J. W. (2002). Pseudooutbreak of Mycobacterium fortuitum due to contaminated ice machines. Am J Infect Control 30, 184-186. 
Lan, R. \& Reeves, P. R. (2001). When does a clone deserve a name? A perspective on bacterial species based on population genetics. Trends Microbiol 9, 419-424.

Luquín, M., Lanéelle, M. A., Ausina, V., Garcia Barceló, M., Belda, F., Alonso, C. \& Prats, G. (1991a). Distribution of a novel mycolic acid in species of the genus Mycobacterium. Int J Syst Bacteriol 41, 390-394.

Luquin, M., Ausina, V., López-Calahorra, F., Belda, F., GarcíaBarceló, M., Celma, C. \& Prats, G. (1991b). Evaluation of practical chromatographic procedures for identification of clinical isolates of mycobacteria. J Clin Microbiol 29, 120-130.

Marks, J. \& Trollope, D. R. (1960). A study of the 'anonymous' mycobacteria. I. Introduction; colonial characteristics and morphology; growth rates; biochemical tests. Tubercle 41, 51-62.

Menendez, M. C., Garcia, M. J., Navarro, M. C., Gonzalez-yMerchand, J. A., Rivera-Gutierrez, S., Garcia-Sanchez, L. \& Cox, R. A. (2002). Characterization of an rRNA operon ( $\mathrm{rrnB}$ ) of $\mathrm{Myco-}$ bacterium fortuitum and other mycobacterial species: implications for the classification of mycobacteria. J Bacteriol 184, 1078-1088.

Muñoz, M., Julian, E., García-Barceló, M., Ausina, V. \& Luquin, M. (1997). Easy differentiation of Mycobacterium mucogenicum from other species of the Mycobacterium fortuitum complex by thin-layer and gas chromatography of fatty esters and alcohols. J Chromatogr B Biomed Sci Appl 689, 341-347.

Raad, I. I., Vartivarian, S., Khan, A. \& Bodey, G. P. (1991). Catheterrelated infections caused by the Mycobacterium fortuitum complex: 15 cases and review. Rev Infect Dis 13, 1120-1125.

Ringuet, H., Akoua-Kofel, C., Honore, S., Varnerot, A., Vincent, V., Berche, P., Gaillard, J. L. \& Pierre-Audigier, C. (1999). hsp65 sequencing for identification of rapidly growing mycobacteria. J Clin Microbiol 37, 852-857.

Schröder, K.-H., Naumann, L., Kroppenstedt, R. M. \& Reischl, U. (1997). Mycobacterium hassiacum sp. nov., a new rapidly growing thermophilic mycobacterium. Int J Syst Bacteriol 47, 86-91.

Silcox, V. A., Good, G. C. \& Floyd, M. M. (1981). Identification of clinically significant Mycobacterium fortuitum complex isolates. J Clin Microbiol 14, 686-691.

Springer, B., Böttger, E. C., Kirschner, P. \& Wallace, R. J., Jr (1995). Phylogeny of the Mycobacterium chelonae-like organism based on partial sequencing of the 16S rRNA gene and proposal of Mycobacterium mucogenicum sp. nov. Int J Syst Bacteriol 45, 262-267.

Telenti, A., Marchesi, F., Balz, M., Bally, F., Böttger, E. C. \& Bodmer, T. (1993). Rapid identification of mycobacteria to the species level by polymerase chain reaction and restriction enzyme analysis. J Clin Microbiol 31, 175-178.

Tsukamura, M. (1967). Identification of mycobacteria. Tubercle 48, 311-338.

Vestal, A. L. (1975). Procedures for the Isolation and Identification of Mycobacteria. CDC publication 76-8230. Atlanta, GA: Centers for Disease Control.

Wayne, L. G. \& Kubica, G. P. (1986). The mycobacteria. In Bergey's Manual of Systematic Bacteriology, vol. 2, pp. 1435-1457. Edited by P. H. A. Sneath, N. S. Mair, M. E. Sharpe \& J. G. Holt. Baltimore: Williams \& Wilkins.

Willumsen, P., Karlson, U., Stackebrandt, E. \& Kroppenstedt, R. M. (2001). Mycobacterium frederiksbergense sp. nov., a novel polycyclic aromatic hydrocarbon-degrading Mycobacterium species. Int J Syst Evol Microbiol 51, 1715-1722.

Wilson, R. W., Steingrube, V. A., Böttger, E. C. \& 11 other authors (2001). Mycobacterium immunogenum sp. nov., a novel species related to Mycobacterium abscessus and associated with clinical disease, pseudo-outbreaks and contaminated metalworking fluids: an international cooperative study on mycobacterial taxonomy. Int J Syst Evol Microbiol 51, 1751-1764. 